\title{
Naa I0p Enhances Chemosensitivity to Cisplatin in Oral Squamous Cell Carcinoma Cells
}

This article was published in the following Dove Press journal:

Cancer Management and Research

\author{
Lichun Sun ${ }^{1} * *$ \\ Kaixin Wang ${ }^{2, *}$ \\ Lu Peng' \\ Jinfang Zhang ${ }^{2}$ \\ Jie Yang $^{3}$ \\ Juan Zhao' \\ Jiang $\mathrm{Xu}{ }^{\prime}$ \\ Jun Zheng' \\ Yan Zeng (D)
}

'Department of Stomatology and Key Laboratory of Xinjiang Endemic and Ethnic Diseases, The First Affiliated Hospital, Shihezi University School of Medicine, Shihezi, Xinjiang, People's Republic of China; ${ }^{2}$ Huazhong University of Science and Technology Union Shenzhen Hospital, Shenzhen, Guangdong, People's Republic of China; ${ }^{3}$ Department of Laboratory, The First Affiliated Hospital, Shihezi University School of Medicine, Shihezi, Xinjiang, People's Republic of China

*These authors contributed equally to this work

\begin{abstract}
Background: This study aimed to investigate the function and underlying molecular mechanism of $\mathrm{N}-\alpha$-acetyltransferase 10 protein (Naa10p) in cisplatin (CDDP) chemosensitivity in oral squamous cell carcinoma (OSCC).

Methods: Salivary Naa10p levels in 76 OSCC patients undergoing CDDP-based chemotherapy were detected using enzyme-linked immunosorbent assay. Quantitative real-time polymerase chain reaction and Western blot were used to examine the expression of Naa10 $p$ in constructed CDDP-resistant OSCC cell (Cal-27/CDDP) lines and nude mouse model. In addition, the tumor volume and weight of nude mice were analyzed. Lentiviral system was employed to establish and identify OSCC cell lines with stable Naa10p interference or overexpression. MTT assay was used for drug sensitivity analysis. P-gp and Bcl-2 expression levels were tested by Western blot.
\end{abstract}

Results: Higher salivary Naa10p expression was present in the complete response/partial response group $(n=46)$ compared to the stable disease/progressive disease group $(n=30)$ in OSCC patients receiving chemotherapy treatment. Naa10p expression was down-regulated in Cal-27/CDDP cells and tissues. Naa10p overexpression significantly reduced the expression level of drug-resistant molecules. Naa10p was related to CDDP resistance and enhanced CDDP sensitivity in OSCC according to drug sensitivity analysis and nude mouse model experiments.

Conclusion: Naa10p plays a tumor suppressor gene role and is associated with CDDP resistance in OSCC. It can enhance CDDP sensitivity in OSCC and may be a potential target for OSCC chemotherapy.

Keywords: $\mathrm{N}$ - $\alpha$-acetyltransferase 10 protein, oral squamous cell carcinoma, cisplatin, chemosensitivity

\section{Introduction}

Oral squamous cell carcinoma (OSCC) is an emerging common oral malignant tumor, comprising almost $3 \%$ of all new clinical cancer cases. ${ }^{1}$ Even though current treatment strategy has made significant progress, the overall five-year survival rate for OSCC patients remains unsatisfactory at $<50 \% .^{2}$ Routine OSCC treatment includes surgery, radiotherapy, and chemotherapy. Surgery combined with chemotherapy and radiotherapy can improve the overall survival, especially for patients with advanced oral cancer. ${ }^{3}$ Nevertheless, drug resistance in chemotherapy has largely hampered the efficacy of comprehensive treatment for OSCC. ${ }^{4}$ Cisplatin (CDDP) is a platinum-based chemotherapy drug with advantages for treatment of multiple cancers. Its clinical utility is frequently restricted by drug resistance and severe side effects. ${ }^{5}$ However, the mechanism of CDDP resistance is extremely
Correspondence: Yan Zeng; Jun Zheng Department of Stomatology \& Key Deratory of Xinjiang Endemic an Ethnic Disease, The First Affiliated Hospital, Shihezi University School of Medicine, Shihezi, Xinjiang, People's Republic of China

Tel +86 I8997732862; +86 I8997737I92

Email zengyan910@gmail.com;

haohaoay@yeah.net
Cancer Management and Research 202|:|3 |843-|85|

1843

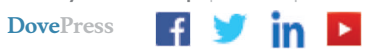

(c) (i) (5) 2021 Sun et al. This work is published and licensed by Dove Medical Press Limited. The full terms of this license are available at https://www.dovepress.com/terms.php (c) BY NC and incorporate the Creative Commons Attribution - Non Commercial (unported, v3.0) License (http:///creativecommons.org/licenses/by-nc/3.0/). By accessing the work you hereby accept the Terms. Non-commercial uses of the work are permitted without any further permission from Dove Medical Press Limited, provided the work is properly attributed. For permission for commercial use of this work, please see paragraphs 4.2 and 5 of our Terms (https://www.dovepress.com/terms.php). 
complex and involves the interaction of many transcription factors, tumor suppressor factors, and related signaling pathways. ${ }^{6}$ Therefore, in-depth knowledge about the molecular mechanism of CDDP resistance in OSCC chemotherapy, screening, and identifying molecular markers related to drug resistance are of great clinical significance for preventing or reversing OSCC drug resistance and improving the therapeutic effect.

$\mathrm{N}$ - $\alpha$-acetyltransferase 10 protein (Naa10p) is the most common catalytic subunit of non-histone N-terminal $\alpha$ acetylase in organisms that takes part in various vital biological processes. ${ }^{7}$ Accumulating evidence has demonstrated that Naa10p is involved in the development of cancers and plays different roles in various tumors. It participates in tumor differentiation, formation, and metastasis in oral, ${ }^{8}$ breast, ${ }^{9,10}$ liver, ${ }^{1,12}$ lung, ${ }^{13}$ prostate, ${ }^{14}$ and colon $^{15}$ cancers. Scholars have indicated that Naa10p is highly expressed in OSCC. Naa10p also has anti-cancer effects on the progression of OSCC. ${ }^{8}$ High Naa10p expression level has a connection with tumor invasiveness and low survival rate in colon cancer patients. ${ }^{16}$ Furthermore, studies involving Naa10p in drug resistance of various cancers have been published. Some researchers have also pointed out that Naa10p can change tumor cell sensitivity to chemotherapeutic drugs by affecting cell apoptosis. ${ }^{1,17}$ However, the molecular mechanism of Naa10p involved in OSCC progression and CDDP chemotherapy resistance remains elusive.

The present study established CDDP-resistant OSCC cell lines and nude mouse models to explore the effect of Naa10p on CDDP chemoresistance in OSCC in order to identify the function of Naa10p in the clinical treatment of OSCC.

\section{Methods}

\section{Study Subjects and Saliva Samples}

The subjects were 76 patients with a clinical diagnosis of OSCC who were treated with CDDP chemotherapy in the First Affiliated Hospital of Shihezi University School of Medicine (Shihezi, China). The patients included 41 males and 35 females with an average age of $62.89 \pm 13.74$ years (range, 22-83 years). The clinical efficacy was evaluated using RECIST guidelines. ${ }^{18}$ Patients with complete response $(\mathrm{CR})$ or partial response $(\mathrm{PR})$ were regarded as responders, and patients with stable disease (SD) or progressive disease (PD) as non-responders. There was no statistically difference between the two groups in terms of age, gender, clinical stage, lymph node metastasis and degree of differentiation.

1-2 mL unstimulated saliva samples were collected from the patients. After centrifuging the saliva samples at $800 \times \mathrm{g}$ (room temperature) for $2 \mathrm{~min}$, the supernatant was transferred to sterile sample tubes, followed by the addition of protease inhibitors. The saliva samples were stored at $-80^{\circ} \mathrm{C}$ for further analysis. Naa10p saliva levels were analyzed using the DL-NAA10-Hu ELISA kit (DL Develop, Kelowna, BC, Canada) according to the manufacturer instructions. This study was approved by the Research Ethics Committee of the First Affiliated Hospital of Medicine College, Shihezi University and was conducted in accordance with the Declaration of Helsinki. All patients provided informed consent.

\section{Cell Culture}

Cal-27 cells were obtained from the American Type Culture Collection (ATCC, Manassas, VA, USA) and cultured with standard culture conditions. In the early stages, low-dose CDDP was used to continuously induce Cal-27 cells. The dose was then gradually increased to treat Cal27 cells until the surviving cells showed a normal growth pattern in order to construct CDDP-resistant OSCC cells (Cal-27/CDDP). All cell lines were kept in moist air with $5 \% \quad \mathrm{CO}_{2}$ at $37^{\circ} \mathrm{C}$ in DMEM containing $10 \%$ FBS (Invitrogen, Carlsbad, CA, USA).

\section{Generation of Stable Naa I0p Knockdown or Overexpression Cell Lines}

Naa10p interference lentivirus (LV-shNaa10p), Naa10p overexpression lentivirus (LV-Naa10p), and control lentivirus (LV-NC) were constructed, packaged, and purified by Shanghai GenePharma Co., Ltd. Cal-27 cells were then infected with LV-shNaa10p, LV-Naa10p, and LV-NC. In addition, different multiplicity of infection (MOI) was established to determine the best infection efficiency. Western blot detected the interference and overexpression efficiency of Naa10p.

\section{Quantitative Real-Time Polymerase Chain Reaction (qRT-PCR)}

Total RNA in OSCC cells was isolated using TRIzol reagent (Invitrogen, Carlsbad, USA) according to manufacturer instructions. PrimeScript ${ }^{\mathrm{TM}}$ RT reagent kit (Takara, Dalian, China) was used to reverse transcribe into cDNA. Naa10p expression levels were quantified 
using SYBR Green qPCR Master Mix (Toyobo, Osaka, Japan). Glyceraldehyde-3-phosphate dehydrogenase (GAPDH) served as an internal reference. Relative Naa10p expression was calculated using the $2^{-\Delta \Delta C T}$ method. Primer sequences were as follows: Naa10p, forward: 5'-ATGAACATCCGCAATG-3', reverse: 5'-AC AATCTTCCCATTCTC-3'; GAPDH, forward: 5'-GC ACCGTCAAGGCTGAGAAC-3'， reverse: 5'-ATGGTG GTGAAGACGCCAG T-3'.

\section{Drug Sensitivity Determination}

The infected OSCC cells were treated with different concentrations of CDDP ranging from 0.1 to $8 \mu \mathrm{g} / \mathrm{mL}$ for 72 h. MTT assay was performed for drug sensitivity analysis. The absorbance of each well at $490 \mathrm{~nm}$ was measured using a microplate reader (Bio-Rad Laboratories, Inc., CA, USA). Half maximal inhibitory concentration $\left(\mathrm{IC}_{50}\right)$ and resistance index (RI) were analyzed to evaluate CDDP-induced cytotoxicity.

\section{Western Blot}

The whole protein samples from OSCC tissues and cells were extracted using a radioimmunoprecipitation assay buffer containing a protease inhibitor (1:100; Sigma, St. Louis). A total of $50 \mu \mathrm{g}$ of lyase were imprinted using $10 \%$ sodium dodecyl sulfate-polyacrylamide gel electrophoresis and then transferred to a polyvinylidene fluoride membrane (Merck Millipore). After incubation with a primary antibody at $4^{\circ} \mathrm{C}$ overnight, the membrane was incubated with a secondary antibody for $1 \mathrm{~h}$ at room temperature. Naa10p, Bcl-2, and P-gp expression in tumor cells was evaluated using a Western blot. Protein band detection was accomplished using enhanced chemiluminescence (Pierce Biotechnology; Rockford, IL, USA). ImageJ software was used for quantification and comparison of protein bands. Band density was standardized to $\beta$ actin.

\section{Xenograft Tumor Model in Nude Mice}

Animal experiments were conducted following the agreement of the Ethics Committee of the First Affiliated Hospital of Shihezi University, and performed in accordance with the National Institutes of Health (NIH) guidelines for Care and Use of Laboratory Animals. Four- to six-week-old BALB/c nude mice were bought from Beijing Vital River Laboratory Animal Technology Co., Ltd. Cal-27/CDDP and Cal-27 cells $\left(1.5 \times 10^{7} / \mathrm{mL}\right)$ were injected subcutaneously into the right abdomen in mice.
After ten days, the mice were injected intraperitoneally with CDDP every two days for 14 days. After drug injection, the tumor volumes were measured every two days and calculated using the formula of volume as width ${ }^{2} \times$ length $\times 0.5$. Mice were euthanized for tumor weight analysis. Tumor growth curves were prepared at the end of the experiment.

\section{Statistical Analyses}

Data were presented as mean \pm standard deviation (SD) or standard error (SE). GraphPad Prism software 8.0 was used for statistical analysis. Student's $t$-test was performed to evaluate the differences in Naa10p expression level, tumor volume, and weight between two groups. Statistical differences in protein levels and $\mathrm{IC}_{50}$ values among multiple groups were analyzed using one-way ANOVA. $P<0.05$ was considered statistically significant.

\section{Results}

\section{Correlation Between Salivary Naa I0p} Level and Clinical Chemotherapy Efficacy in OSCC Patients Treated with CDDP

\section{Chemotherapy}

Of all OSCC patients, 46 patients were assessed as chemotherapy responders $(\mathrm{CR}+\mathrm{PR})$ and 30 patients as non-responders $(\mathrm{SD}+\mathrm{PD})$. Salivary Naa10p level was significantly higher in the $\mathrm{PR} / \mathrm{CR}$ group compared to the $\mathrm{PD} / \mathrm{SD}$ group $(P<0.05$; Figure 1), suggesting that Naa10p is associated with OSCC CDDP chemotherapy.

\section{Naa I0p Expression in OSCC Cells and CDDP-Resistant OSCC Cell Lines}

To explore the effect of Naa10p on CDDP resistance in OSCC cells, Naa10p expression in Cal-27/CDDP cells was analyzed using Western blot and qRT-PCR. The findings illustrated that the level of Naa10p expression was remarkably lower in Cal-27/CDDP cells compared to the corresponding parental cells $(P<0.01$; Figure 2$)$. This indicated that Naa10p might be related to CDDP resistance in OSCC.

\section{Determination of Lentivirus Infection Efficiency}

The cells in Naa10p interference, Naa10p overexpression, and control groups infected with lentivirus vector were observed under a confocal laser scanning microscope. The lentivirus infection efficiency was $>90 \%$ with MOI 200, 


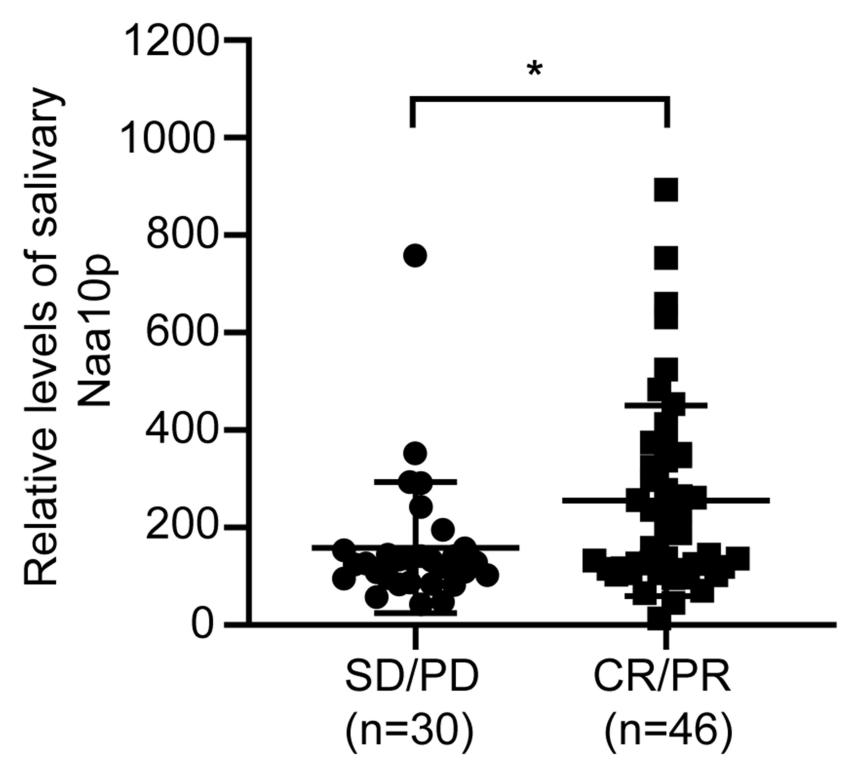

Figure I NaalOp expression in saliva of OSCC patients treated with cisplatin chemotherapy. $* P<0.05$.

Abbreviations: CR, complete remission; PR, partial remission; SD, stability disease; PD, progressive disease.

which was then selected for cell infection. Western blot analysis identified the efficiency of the lentivirus infection. Naa10p expression was reduced in the LV-shNaa10p group and increased in the LV-Naa10p group (Figure 3).

\section{Growth Inhibitory Effect of Different CDDP Concentrations on Cal-27 Cells}

The proliferation inhibition effect of CDDP on Cal-27 cells was enhanced by increasing drug concentration. The growth inhibition rate of Naa10p knockdown cells was significantly lower than that of control cells. Conversely, Naa10p overexpression significantly enhanced the growth inhibition of
CDDP in Cal-27 cells (Figure 4A). The effect of Naa10p expression level on CDDP sensitivity was evaluated according to the $\mathrm{IC}_{50}$ value and $\mathrm{RI}$ (Table 1 and Figure 4B). The $\mathrm{IC}_{50}$ value and $\mathrm{RI}$ for CDDP in the $\mathrm{LV}$-shNaa10p group were higher compared to the control $(P<0.05)$, indicating that interfering with Naa10p can reduce CDDP sensitivity in Cal-27 cells and signifying that OSCC cell drug resistance was enhanced. Cal-27 cell sensitivity to CDDP was significantly enhanced, while $\mathrm{IC}_{50}$ value and $\mathrm{RI}$ were obviously reduced in the LV-Naa10p group $(P<0.05)$.

\section{Drug-Resistant Molecule $\mathrm{Bcl}-2$ and P-gP Expression in OSCC Cells is Affected by Naa I0p Expression Changes}

Western blot analysis evaluated the expression of drug resistance-related molecules Bcl-2 and P-gp in different groups. The results showed that interfering with Naa10p markedly enhanced P-gp and Bcl-2 expression levels, while upregulation of Naa10p significantly inhibited Bcl-2 and P-gp expression levels (Figure 5). These results suggested that Naa10p is closely related to CDDP chemotherapy resistance.

\section{Relationship Between Naa IOp and CDDP-Resistant OSCC Cells in vivo}

The present study demonstrated that Naa10p enhances OSCC cell sensitivity to CDDP in vitro. For further confirmation of the Naa10p effect on CDDP chemoresistance, a CDDP-resistant tumor model using nude mice was constructed. The tumor volume of nude mice in the Cal-27/CDDP and control groups was calculated to generate a plot of tumor volume and weight changes. Compared to the control group, both tumor

B
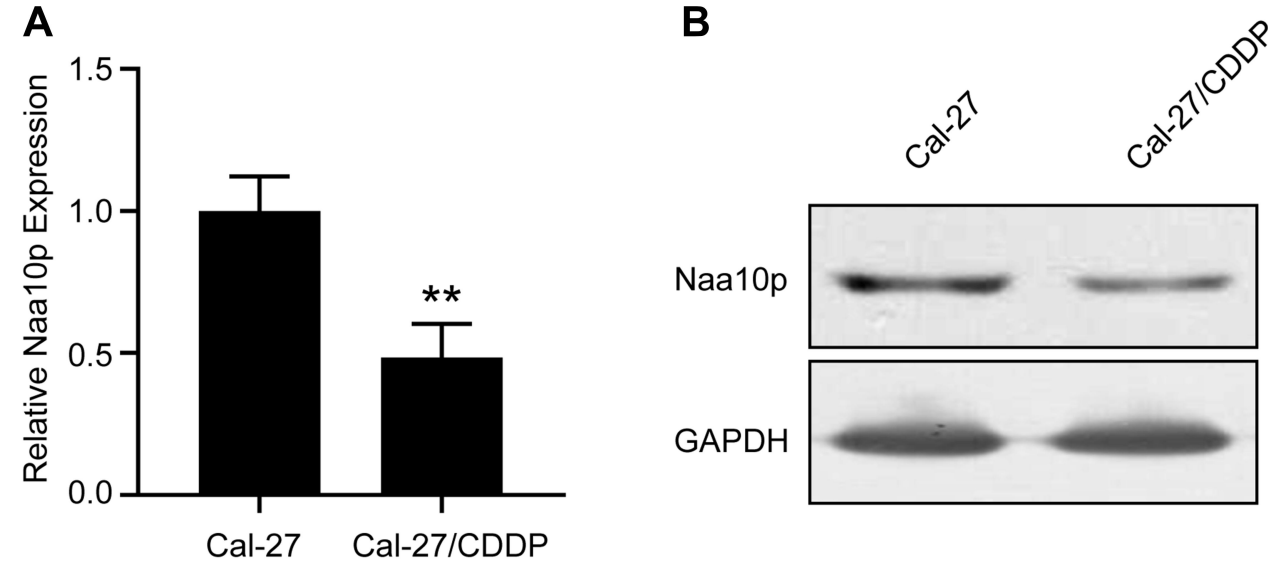

Figure 2 Naa I0p expression in CDDP-resistant OSCC cells. (A) qPCR was performed to analyze Naa I0p expression in CDDP-resistant cells and control cells. **P < 0.0 I. (B) Western blot was performed to analyze Naa IOp expression in CDDP-resistant cells and control cells. 
A

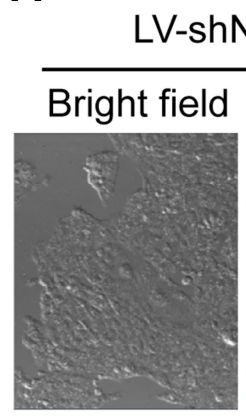

V-shNaa10p

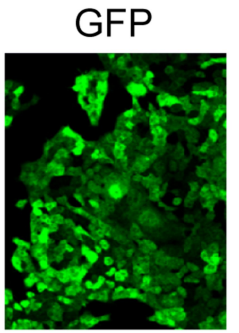

LV-Naa10p

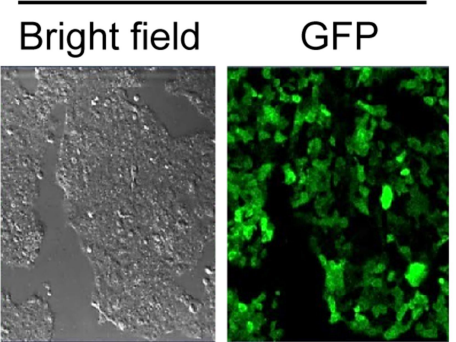

B

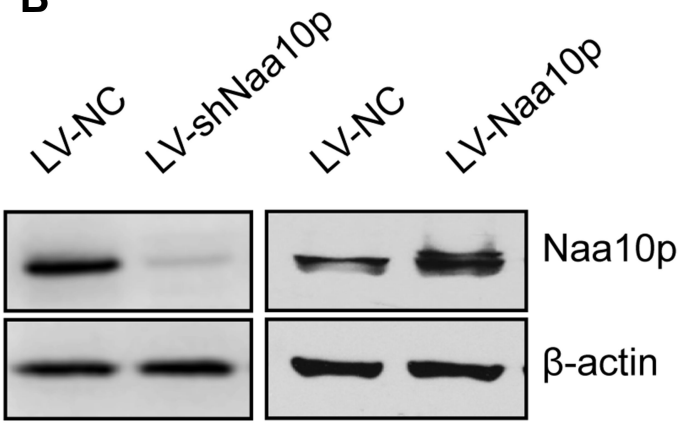

Figure 3 Determination of lentivirus infection efficiency. (A) Infection efficiency of lentivirus in Cal-27 cells with MOI 200 was detected under the laser confocal microscope (×40). (B) Efficiency identification of interference or overexpression of NaalOp by lentivirus infection.

A

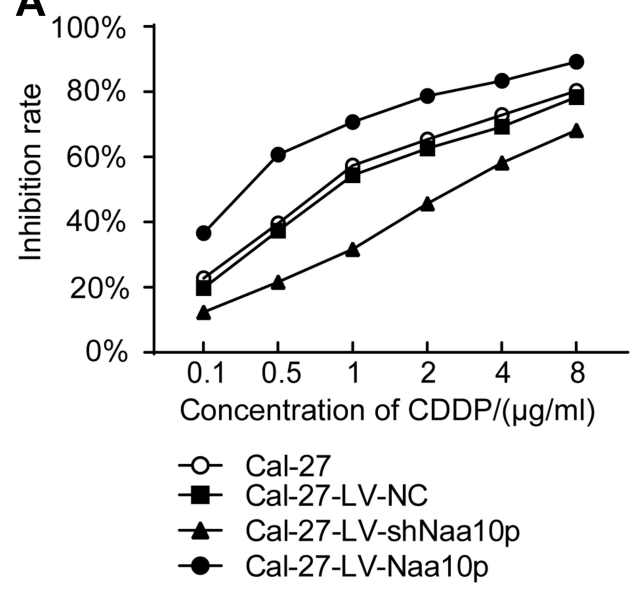

B

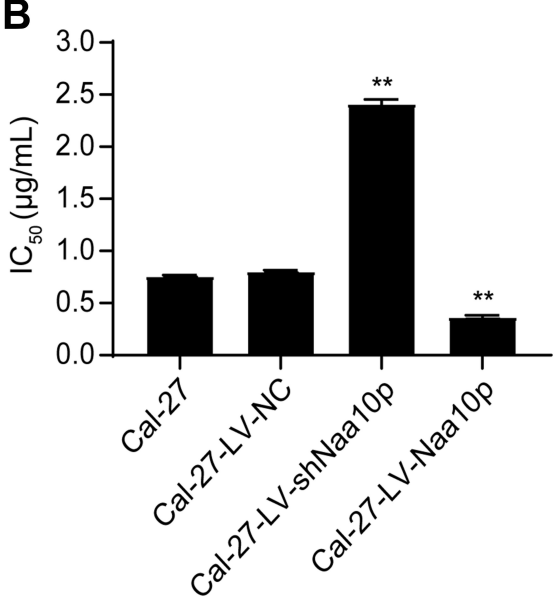

Figure 4 Effects of different concentrations of cisplatin on Cal-27 cells. (A) Cell growth inhibitory effect of cisplatin on each group. (B) IC 50 values of cisplatin in each group. $* * P<0.01$ vs $\mathrm{Cal}-27$ group.

weight and volume were greater in the Cal-27/CDDP group $(P<0.05$; Figure 6$)$. Western blot analysis was used to examine Naa10p expression in tumor tissues of four nude mice in the two groups. Results revealed that Naa10p expression levels in tumor tissues of CDDPresistant nude mice were remarkably downregulated in comparison to the control group $(P<0.05)$. All of these data indicated that Naa10p is related to CDDP resistance in OSCC and enhances CDDP sensitivity in OSCC.

Table I CDDP Sensitivity of Cal-27 Cells with Different Levels of NaalOp

\begin{tabular}{|l|c|c|c|}
\hline Group & IC $_{\mathbf{5 0}}(\mu \mathrm{g} / \mathbf{m L})$ & $\mathbf{R I}$ & $\boldsymbol{P}$ value \\
\hline Cal-27 & $0.759 \pm 0.009$ & - & - \\
Cal-27-LV-NC & $0.803 \pm 0.013$ & 1.014 & 0.372 \\
Cal-27-LV-shNaa I0p & $2.412 \pm 0.04 \mathrm{I}$ & 3.042 & 0.000 \\
Cal-27-LV-Naa Ip & $0.366 \pm 0.018$ & 0.457 & 0.000 \\
\hline
\end{tabular}

\section{Discussion}

The emergence of drug resistance in tumor cells is not only a significant obstacle to successful tumor treatment, but also an important reason for chemotherapy failure. CDDP is a basic chemotherapeutic drug used for clinical treatment of OSCC. However, its anti-tumor effect is seriously limited by tumor cell drug resistance. In the past, it was believed that tumor drug resistance to CDDP was related to the following factors: a decrease or excretion of CDDP by tumor cells, change in the activity of the target protein, enhancement of the cells' ability to repair DNA, acceleration of DNA adduct decomposition, and change of some regulatory factors' expression in the apoptosis signaling transduction pathway. ${ }^{19}$

The effect of Naa10p on different tumors is controversial. Previous studies have shown that Naa10p is highly expressed in colon cancer, while Naa10p level upregulation is associated with poor prognosis. ${ }^{15}$ Naa10p deletion induces colon cancer cell apoptosis by RelA/p65 regulation 
A
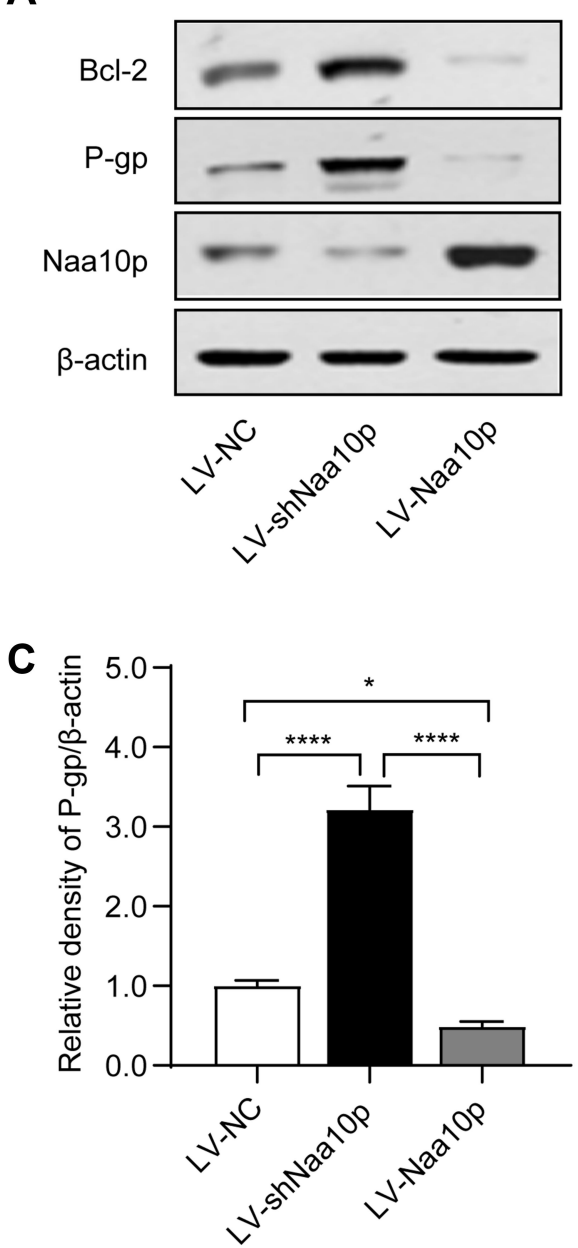

B
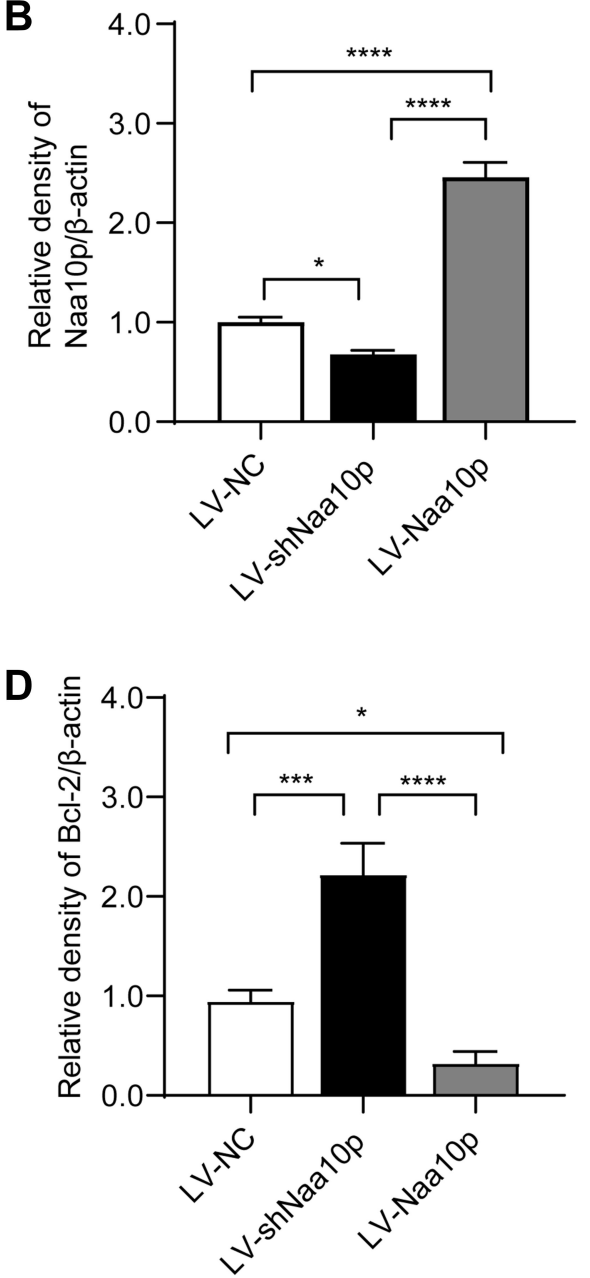

Figure 5 Drug-resistant molecules expression of Cal-27 cells with different expression levels of Naa l0p. (A) The expression of Naa I0p, P-gp and Bcl-2 in different groups were detected by Western blot. (B) Relative density of Naa I0p normalized to $\beta$-actin. (C) Relative density of P-gP normalized to $\beta$-actin. (D) Relative density of Bcl-2 normalized to $\beta$-actin. $* P<0.05$, $* * * p<0.001$, $* * * * p<0.0001$.

of MCL1 expression. ${ }^{7}$ In addition, high Naa10p expression in hepatocellular carcinoma is related to its recurrence and poor patient survival. ${ }^{12}$ In vitro studies have revealed that Naa10p endows it with carcinogenicity through SAMHD1 acetylation. ${ }^{11}$ These results tend to support tumor growth promotion by Naa10p. However, Naa10p has been demonstrated to facilitate DNA damage-mediated apoptosis as well. ${ }^{20}$ Naa10p stabilizes TSC2 through acetylation and inhibits mTOR activity, thereby inhibiting cell proliferation and tumor growth. ${ }^{21}$ In addition, Naa10p restrains tumor cell metastasis in breast and lung cancers. ${ }^{9}$ These study results indicate that Naa10p exerts an anti-cancer effect on tumors. These contradictory experimental data may not only be attributed to the differences in experimental approach and materials, but also suggest that Naa10p plays a diverse role in different tumor types and stages. Our previous studies have revealed that salivary Naa10p is highly expressed in OSCC patients. The level of Naa10p in saliva was associated with OSCC differentiation and lymph node metastasis. ${ }^{22}$ Following up on OSCC patients treated with CDDP, we found that high Naa10p saliva levels were associated with better disease control. This indicated that Naa10p expression level might be correlated with CDDP chemotherapy. It has been reported that Naa10p may have a function in DNA repair because RAD51 expression is down-regulated after Naa10p interference. RAD51 is a member of the DNA repair complex, which plays a critical role in repairing double-strand DNA damage. ${ }^{23}$ There is growing evidence that Naa10p is connected to drug resistance in tumor cells and plays a major role in the process of drug resistance. ${ }^{1,17}$ So far, however, the mechanism of Naa10p CDDP resistance in OSCC has not been clearly described. We speculate that Naa10p level is correlated with CDDP resistance in OSCC. 


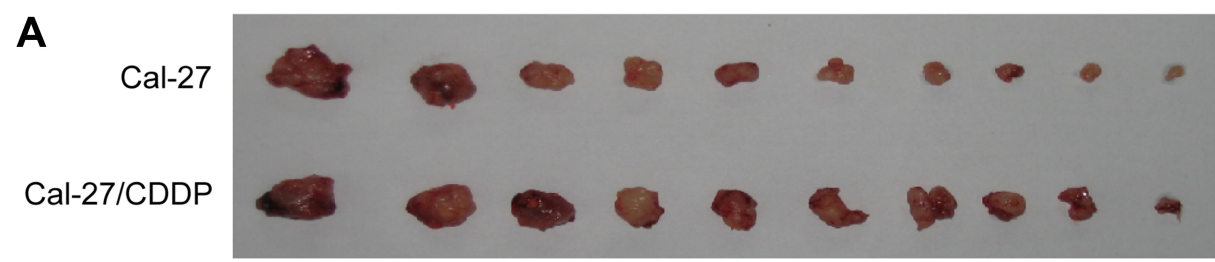

B

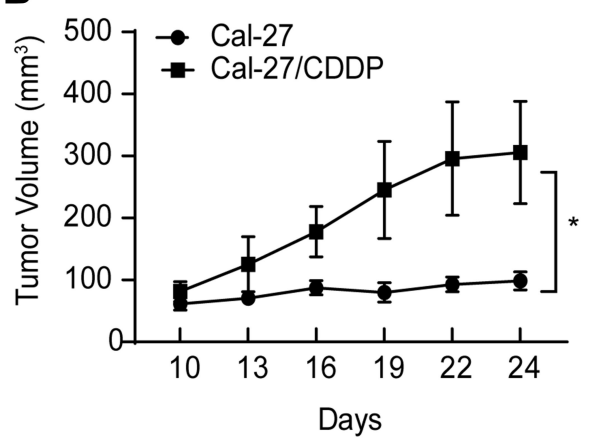

D

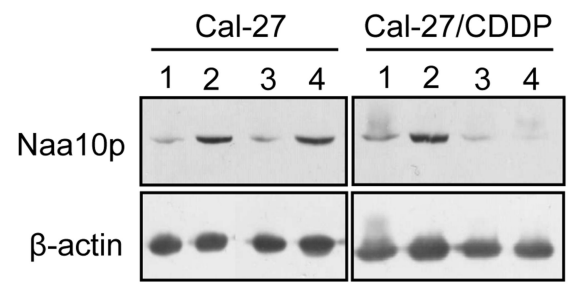

C

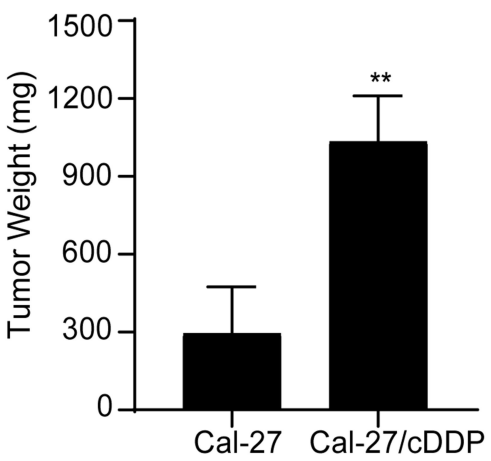

E

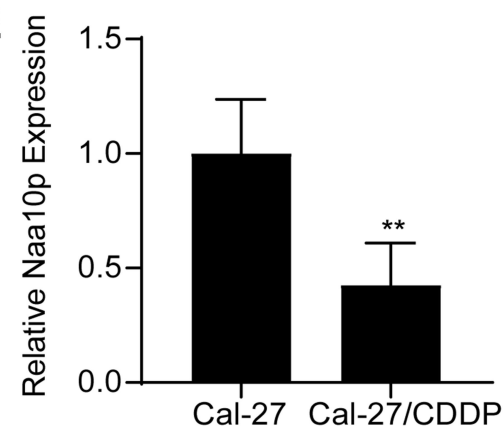

Figure 6 Relation between Naa I0p and CDDP-resistant OSCC cells in mice model. (A) Images of OSCC xenograft tumors. (B) Tumor volume growth curves. (C) Tumor weights were analyzed. ( $D$ and $\mathbf{E}$ ) Naa IOp expression in tumor tissues. $* P<0.05$, $* * p<0.01$.

In order to verify this hypothesis, the present study established CDDP-resistant OSCC cell lines in vitro. The data indicated that Naa10p expression is markedly reduced in Cal-27/CDDP cells compared to normal Cal-27 cells. Studies have further shown that there is a correlation between Naa10p and CDDP chemotherapy resistance. In addition, we successfully constructed OSCC cell lines with a stable interference and overexpression of Naa10p and a corresponding control cell line using a lentivirus system. The MTT assay was employed to analyze the growth inhibitory effect of different CDDP concentrations in each treatment group of cells. After the CDDP treatment, the $\mathrm{IC}_{50}$ value for the interference Naa10p cells was higher than the $\mathrm{IC}_{50}$ value for the control cells, indicating that interference with Naa10p can reduce the sensitivity of Cal27 cells to CDDP, meaning enhanced drug resistance of
Cal-27 cells. The $\mathrm{IC}_{50}$ value for cells in the Naa10p overexpression group decreased significantly, indicating that Naa10p can enhance the sensitivity of Cal-27 cells to CDDP chemotherapy, which weakens drug resistance.

To further clarify the relationship between Naa10p and CDDP chemotherapy, the expression levels of drug resistance-related molecules Bcl-2 and P-gp were measured in all treatment groups. The data showed that Naa10p interference increased the expression level of drug resistance molecules in Cal-27 cells. Conversely, up-regulating Naa10p significantly decreased the expression level of drug resistance molecules, indicating that Naa10p is closely related to CDDP chemotherapy resistance. Apoptosis induction is the main method for CDDP to exert cytotoxicity. ${ }^{24}$ Apoptosis failure is the main mechanism of CDDP chemotherapy resistance. ${ }^{25}$ The apoptotic 
pathway can be regulated by apoptotic factors. The antiapoptotic protein Bcl-2 can activate caspase- 3 and -9 by inhibiting the release of cytochrome $\mathrm{c}$ from the mitochondria into the cytosol, thereby inhibiting cell apoptosis. ${ }^{26}$ Studies have reported that Bcl-2 protects normal cells from toxicity, promotes cell survival and cell arrest, and enhances cytotoxicity of CDDP chemotherapy and drug resistance of tumor cells by upregulating p27 and p130. ${ }^{27,28}$ In this study, a decrease in anti-apoptotic factor Bcl-2 in the Naa10p overexpression cells suggested that Naa10p can promote apoptosis and increase the sensitivity of Cal-27 cells to CDDP. This indicates that Naa10p has a significant inhibitory effect on apoptosis induced by CDDP, which leads to the reduction of therapeutic CDDP cytotoxicity. P-gp is a product of multi-drug resistance gen-1. After it is combined with chemotherapeutic drugs, it uses the energy provided by ATP to combine with ATP to pump the intracellular chemotherapeutic drugs out of the cell against the concentration gradient, thereby reducing the cumulative concentration of the drugs and hindering the chemotherapeutic drug efficacy. ${ }^{29} \mathrm{P}$-gp expression has been positively correlated with the observed drug resistance. ${ }^{28} \mathrm{~A}$ decrease in P-gp in Cal-27 cells overexpressing Naa10p suggested that Naa10p may reduce the number of drug pumps on the cell membrane, thus increasing the concentration of CDDP in tumor cells and enhancing CDDP sensitivity in Cal-27 cells.

The present study established a transplanted tumor model in nude mice with CDDP-resistant OSCC cell lines and control cells as a tool for Naa10p to mediate CDPP resistance in vivo. The results showed that CDDP had a significant inhibitory effect on nude mouse OSCC xenografts, while the inhibitory effect of CDDP on CDDPresistant nude OSCC mice was weakened. Moreover, the Naa10p level in tumor tissues of the Cal-27/CDDP group was significantly decreased, which further indicated that Naa10p is related to CDDP resistance in OSCC and enhances OSCC cell sensitivity to CDDP chemotherapy. These results are largely consistent with the in vitro experiments.

The above findings indicate that Naa10p may serve as a tumor suppressor in OSCC. This is consistent with the previously reported results. ${ }^{8}$ Moreover, the present work indicated that Naa10p participates in CDDP chemoresistance in OSCC. Researchers have found that in cervical cancer cells, down-regulation of Naa10p expression can promote apoptosis and increase cell sensitivity to the chemotherapeutic drug daunorubicin. ${ }^{17}$ It was also reported that overexpression of Naa10p and its acetylation combined with RIP1 can significantly enhance activation of $\mathrm{NF}-\kappa \mathrm{B}$ induced by adriamycin and promote cell apoptosis. ${ }^{30}$ However, CDDP resistance is related to the mechanism of reducing intracellular CDDP accumulation and inhibiting CDDP-induced apoptosis through overactivation of NF- $\kappa B .{ }^{31}$ Activated NF- $\mathrm{KB}$ entering the nucleus can promote the transcription of $\mathrm{Bcl}-2$ to inhibit apoptosis. Moreover, Naa10p inhibits apoptosis by activating the NF$\kappa \mathrm{B}$ pathway. ${ }^{7} \mathrm{Bcl}-2$ in OSCC cells had a low expression after up-regulating Naa10p in the present study, indicating that Naa10p may activate NF- $\mathrm{kB}$ to promote apoptosis and increase CDDP sensitivity in OSCC cells. This contradicts previous results and may be related to the $\mathrm{N}$-terminal amino acid acetylation mediated by Naa10p. Its specific mechanism needs further study.

In conclusion, the present study determined that the high salivary Naa10p level in OSCC patients is associated with a significant CDDP chemotherapy effect, and that Naa10p overexpression can enhance CDDP sensitivity in OSCC cells. Conversely, Naa10p knockdown reduced CDDP sensitivity in OSCC cells. Nude mouse xenograft model experiments further confirmed that Naa10p increases CDDP chemosensitivity in OSCC cells. These findings provide an experimental foundation for the study of the later mechanism stages of Naa10p CDDP resistance in OSCC.

\section{Acknowledgments}

The National Natural Science Foundation of China (81560473, 81560442), Xinjiang Production and Construction Corps Key Areas Innovation Team Project (2018CB002), and the foundation of Shihezi University (CGZH202001) funded this study.

\section{Disclosure}

All authors declare that there are no conflicts of interest in this work.

\section{References}

1. Brocklehurst PR, Baker SR. Speight PM Oral cancer screening: what have we learnt and what is there still to achieve? Future Oncol. 2010;6:299-304. doi:10.2217/fon.09.163

2. Kessler P, Grabenbauer G, Leher A, et al. Neoadjuvant and adjuvant therapy in patients with oral squamous cell carcinoma Long-term survival in a prospective, non-randomized study. $\mathrm{Br} J$ Oral Maxillofac Surg. 2008;46:1-5. doi:10.1016/j.bjoms.2007.08.006

3. Furness S, Glenny AM, Worthington HV, et al. Interventions for the treatment of oral cavity and oropharyngeal cancer: chemotherapy. Cochrane Database Syst Rev. 2010;4:CD006386. 
4. Daniela DSS, Michael H, Alex M, et al. Recurrent oral cancer: current and emerging therapeutic approaches. Front Pharmacol. 2012;3:149.

5. Florea AM, Büsselberg D. Cisplatin as an anti-tumor drug: cellular mechanisms of activity, drug resistance and induced side effects. Cancers (Basel). 2011;3:1351-1371. doi:10.3390/cancers3011351

6. Galluzzi L, Senovilla L, Vitale I, et al. Molecular mechanisms of cisplatin resistance. Oncogene. 2012;31:1869-1883. doi:10.1038/ onc. 2011.384

7. Xu H, Jiang B, Meng L, et al. N- $\alpha$-acetyltransferase 10 protein inhibits apoptosis through RelA/p65-regulated MCL1 expression. Carcinogenesis. 2012;33:1193-1202. doi:10.1093/carcin/bgs144

8. Zeng Y, Zheng J, Zhao J, et al. High expression of Naa10p associates with lymph node metastasis and predicts favorable prognosis of oral squamous cell carcinoma. Tumour Biol. 2016;37:6719-6728. doi:10.1007/s13277-015-4563-Z

9. Zeng Y, Min L, Han Y, et al. Inhibition of STAT5a by Naa10p contributes to decreased breast cancer metastasis. Carcinogenesis. 2014;35:2244-2253. doi:10.1093/carcin/bgu132

10. Zhang Y, Zhou H, Tao Y, et al. ARD1 contributes to IKK $\beta$-mediated breast cancer tumorigenesis. Cell Death Dis. 2018;9:860. doi:10.1038/s41419-018-0921-2

11. Lee EJ, Seo JH, Park JH, et al. SAMHD1 acetylation enhances its deoxynucleotide triphosphohydrolase activity and promotes cancer cell proliferation. Oncotarget. 2017;8:68517-68529. doi:10.18632/ oncotarget.19704

12. Lee D, Jang MK, Seo JH, et al. ARD1/NAA10 in hepatocellular carcinoma: pathways and clinical implications. Exp Mol Med. 2018;50:1-12. doi:10.1038/s12276-018-0187-x

13. Hua KT, Tan CT, Johansson G, et al. N- $\alpha$-acetyltransferase 10 protein suppresses cancer cell metastasis by binding PIX proteins and inhibiting Cdc42/Rac1 activity. Cancer Cell. 2011;19:218-231. doi:10.1016/j.ccr.2010.11.010

14. Kuhns KJ, Zhang G, Wang Z, et al. ARD1/NAA10 acetylation in prostate cancer. Exp Mol Med. 2018;50:1-8. doi:10.1038/s12276018-0107-0

15. Yang H, Li Q, Niu J, et al. microRNA-342-5p and miR-608 inhibit colon cancer tumorigenesis by targeting NAA10. Oncotarget. 2016;7:2709-2720. doi:10.18632/oncotarget.6458

16. Jiang B, Ren T, Dong B, et al. Peptide mimic isolated by autoantibody reveals human arrest defective 1 overexpression is associated with poor prognosis for colon cancer patients. Am J Pathol. 2010;177:1095-1103. doi:10.2353/ajpath.2010.091178

17. Arnesen T, Gromyko D, Pendino F, et al. Induction of apoptosis in human cells by RNAi-mediated knockdown of hARD1 and NATH, components of the protein $\mathrm{N}$-alpha-acetyltransferase complex. Oncogene. 2006;25:4350-4360. doi:10.1038/sj.onc.1209469
18. Therasse P, Arbuck SG, Eisenhauer EA, et al. New guidelines to evaluate the response to treatment in solid tumors. European organization for research and treatment of cancer, National Cancer Institute of the United States, National Cancer Institute of Canada. J Natl Cancer Inst. 2000;92:205-216. doi:10.1093/jnci/92.3.205

19. Wernyj RP, Morin PJ. Molecular mechanisms of platinum resistance: still searching for the Achilles' heel. Drug Resist Updat. 2004;7:227-232. doi:10.1016/j.drup.2004.08.002

20. Kalvik TV. Arnesen T Protein N-terminal acetyltransferases in cancer. Oncogene. 2013;32:269-276. doi:10.1038/onc.2012.82

21. Kuo HP, Lee DF, Chen CT, et al. ARD1 stabilization of TSC2 suppresses tumorigenesis through the mTOR signaling pathway. Sci Signal. 2010;3:ra9. doi:10.1126/scisignal.2000590

22. Zheng J, Sun L, Yuan W, et al. Clinical value of Naa10p and CEA levels in saliva and serum for diagnosis of oral squamous cell carcinoma. J Oral Pathol Med. 2018;47:830-835. doi:10.1111/ jop. 12767

23. Fisher TS, Etages SD, Hayes L, et al. Analysis of ARD1 function in hypoxia response using retroviral RNA interference. J Biol Chem. 2005;280:17749-17757. doi:10.1074/jbc.M412055200

24. Nagelkerke A, Mujcic H, Bussink J, et al. Hypoxic regulation and prognostic value of LAMP3 expression in breast cancer. Cancer. 2011;117:3670-3681. doi:10.1002/cncr.25938

25. Brabec V, Kasparkova J. Molecular aspects of resistance to antitumor platinum drugs. Drug Resist Updat. 2002;5:147-161. doi:10.1016/ S1368-7646(02)00047-X

26. Zheng HC. The molecular mechanisms of chemoresistance in cancers. Oncotarget. 2017;8:59950-59964.

27. Hatok J, Racay P. Bcl-2 family proteins: master regulators of cell survival. Biomol Concepts. 2016;7:259-270.

28. Lu D, Shi HC, Wang ZX, et al. Multidrug resistance-associated biomarkers PGP, GST-pi, Topo-II and LRP as prognostic factors in primary ovarian carcinoma. Br J Biomed Sci. 2011;68:69-74. doi:10.1080/09674845.2011.11730326

29. van Eerd JE, de Geus-oei LF, Oyen WJ, et al. Scintigraphic imaging of P-glycoprotein expression with a radiolabelled antibody. Eur J Nucl Med Mol Imaging. 2006;33:1266-1272. doi:10.1007/s00259006-0152-0

30. Park J, Kanayama A, Yamamoto K, et al. ARD1 binding to RIP1 mediates doxorubicin-induced NF- $\kappa \mathrm{B}$ activation. Biochem Biophys Res Commun. 2012;422:291-297. doi:10.1016/j.bbrc.2012.04.150

31. Suenaga N, Kuramitsu M, Komure K, et al. Loss of tumor suppressor CYLD expression triggers cisplatin resistance in oral squamous cell carcinoma. Int J Mol Sci. 2019;20:5194. doi:10.3390/ijms20205194
Cancer Management and Research

\section{Publish your work in this journal}

Cancer Management and Research is an international, peer-reviewed open access journal focusing on cancer research and the optimal use of preventative and integrated treatment interventions to achieve improved outcomes, enhanced survival and quality of life for the cancer patient.
The manuscript management system is completely online and includes a very quick and fair peer-review system, which is all easy to use. Visit http://www.dovepress.com/testimonials.php to read real quotes from published authors. 